\title{
Patient-Centered Care and Patient-Reported Measures: Let's Look Before We Leap
}

\author{
Daniel Miller • Carolyn Steele Gray • \\ Kerry Kuluski · Cheryl Cott
}

Published online: 30 October 2014

(C) The Author(s) 2014. This article is published with open access at Springerlink.com

\begin{abstract}
This commentary focuses on patient-reported measures as tools to support patient-centered care for patients with multiple chronic conditions (MCCs). We argue that those using patient-reported measures in care management or evaluation of services for MCC patients should do so in recognition of the challenges involved in treating them. MCC patient care is challenging because (1) it is difficult to specify the causes of particular symptoms; (2) assessment of many important symptoms relies on subjective report; and (3) patients require care from a variety of providers. Due to the multiple domains of health affected in single individuals, and the large variation in needs, care that is holistic and individualized (i.e. patientcentered) is appropriate for MCC patients. However, due to the afore-mentioned challenges, it is important to carefully consider what this care entails and how practical contexts shape it. Patient-centered care for MCC patients implies continuous, dialogic patient-provider relationships, and the formulation of coherent and adaptive multi-disciplinary
\end{abstract}

D. Miller $(\varangle) \cdot$ C. Steele Gray · K. Kuluski

Bridgepoint Collaboratory for Research and Innovation,

Bridgepoint Active Healthcare, 14 St. Matthews Rd.,

Toronto, ON M4M 2B5, Canada

e-mail: dmiller@bridgepointhealth.ca

C. Steele Gray

Health System Performance Research Network, University of Toronto, 155 College Street, 4th floor, Toronto, ON M5T 3M6, Canada

\section{K. Kuluski}

Institute of Health Policy, Management and Evaluation, University of Toronto, Toronto, ON, Canada

\section{Cott}

Department of Physical Therapy, University of Toronto, 500 University Avenue, 8th floor, Toronto, ON M4M 2B5 2, Canada care protocols. We identify two broadly defined contextual influences on the nature and quality of these processes and their outputs: (1) busy practice settings and (2) fragmented information technology. We then identify several consequences that may result from inattention to these contextual influences upon introduction of patient-reported measure applications. To maximize the benefits, and minimize the harms of patient-reported measure use, we encourage policy makers and providers to attend carefully to these and other important contextual factors before, during and after the introduction of patient-reported measure initiatives.

\section{Introduction}

In this commentary, we discuss the use of patient-reported measures and consider how this use may influence the provision of patient-centered care for patients with multiple chronic conditions (MCCs). Patient-reported measures are potentially useful as a means of incorporating patient perspectives and experiences into healthcare decision making. However, since MCC patient health status is highly variable, between individuals and over time [1], the use of standardized patient-report to support better care requires careful consideration. In using these measures to influence care, it is important to recognize the complex challenges associated with understanding and treating patients with MCC and to consider how patient-report applications, once initiated, stand to interact with these challenges to influence care. The objective of this paper is to encourage practitioners, policy makers, and evaluators considering the use of patient-reported measures to think critically about how their use may impact care in unanticipated ways.

We begin by defining MCC, explaining its significance, and specifying the complex challenges involved in 
understanding and addressing the needs of MCC patients. We then provide a general definition of patient-centered care, describe its value for MCC patients, and specify characteristics of a patient-centered approach for treating these patients. Subsequently, we describe the basic types of patient-reported measures, how they are used, and the patient-centered rationale for their use. Finally, we examine patient-reported measures in the context of care provision, and consider the possible influence of their use on care for patients with MCCs, within these contexts.

\section{Multiple Chronic Conditions (MCCs): Prevalence and Significance}

As implied by the term, individuals with MCCs have two or more chronic health conditions, such as cancer, heart disease, diabetes, or depression $[2,3]$. The phenomenon of MCC health status is a significant issue for several reasons. While healthcare has traditionally focused on the needs of single-disease patients, increasing evidence suggests that MCCs may be becoming the norm, rather than the exception [4-6]. One-third of all Canadians with a chronic condition have at least two [1]. As many as $70 \%$ of patients aged $>45$ years have MCCs. Moreover, the odds of having MCCs are significantly greater among older people, suggesting that, as our population ages, so too will the proportion of individuals with more than one condition [3]. The presence of multiple conditions is an independent predictor of low quality of life, mortality, disability, and treatment complications. MCC patients account for a disproportionate amount of healthcare costs and use [4]. For all of these reasons, understanding and providing for the needs of these patients may be one of the most important tasks in contemporary healthcare.

\subsection{MCCs: Care Challenges}

While it is important to address the needs of MCC patients, several challenges make this difficult. MCC patients have multiple needs that require assessment and care from multiple providers [4], who need to integrate diverse perspectives, information, and interventions. Since their conditions are often incurable, measuring illness and monitoring progress may require incorporation of subjective health domains [1] that cannot be directly observed by providers [7-9]. It is also difficult to establish the causes of particular complaints when multiple conditions are present. Providers need to disentangle various potentially interacting symptoms of diverse origin, which makes treatment decisions less straightforward and their effects harder to predict [1]. Finally, since case mixes and populations have high patient-to-patient variability, standardized protocols and disease-specific treatment guidelines are of limited applicability $[1,4]$.

\section{Patient-Centered Care: A Response to the Care Challenges of MCC Patients}

One approach that may be promising for understanding and addressing the needs of MCC patients is patient-centered care $[1,4]$. Patient-centered care refers, in general, to the organization and provision of care around patients' holistic needs, perspectives, experiences, and preferences $[4,10$, 11]. Insofar as it emphasizes individualized holistic care, it is appropriate for the care of patients with multiple conditions due to the high patient-to-patient variability and the multiplicity of each individual's needs [4].

However, attempts to foster a patient-centered approach for MCC patients need to be based on careful consideration of what this is likely to entail. Patient-centered care for these patients implies the assessment of each individual in recognition of the complexity, subjectivity, and changing nature of their health status [1]. It also implies the linking together of multiple episodes of care offered by diverse providers into continuous, integrated care trajectories unique to particular patients [4].

Patient-centered care for patients with MCCs should be understood as a set of processes occurring in the interactions between patient and provider, and between multiple providers. (1) At the direct (patient-provider) care level, taking a patient-centered approach with these patients implies some degree of ongoing patient-provider dialogue, occurring in a continuous relationship [12,13]. (2) At the level of health systems (provider-provider), we argue that patient-centered care implies the ongoing development and adjustment of individualized, coherent but adaptive multidisciplinary care protocols.

\subsection{Direct Care Level (Patient-Provider)}

Stewart et al. propose a version of patient-centered care in which primary care providers work with patients in a continuous, dialogic partnership [12,13]. Consultations are characterized by open dialogue, elicitation of patient priorities and interpretations, and exploration of the subjective and complex aspects of illness. This approach is appropriate for patients with MCCs because of the subjective, complex, and changing nature of their needs [1]. Qualitative dialogue allows the identification of complex relationships between various dimensions of health, causes, and implications that may not be anticipated by highly structured consultation methods [14]. Provider-specific continuity, at least within primary care, is appropriate because patients' health status, already hard to understand 
and predict, changes over time. A continuous relationship allows the dialogic, exploratory element of a single consultation to continue, allowing patient and provider to learn from and respond to changes in health status over a person's life-course.

\subsection{Health System Level (Provider-Provider)}

Where patients have multiple conditions, they often need several types of care from multiple types of providers who work in different organizations. Hence, multiple care episodes need to be integrated not only over time but also across networks of diverse providers and the organizations supporting them (health systems) [4, 11]. However, since MCC populations and case-mixes have high patient-topatient variability, the specific contents and episodic sequences of care also need to be highly variable. Rather than a static model of care, patient-centered care in this context implies a dynamic, adaptive, and interactive process of configuring the components of a health system around individuals' multiple needs. Providers need to collectively participate in the development of tentative pictures of individual patient needs and to adapt or replace these pictures as needs change or information about them changes $[15,16]$.

\section{Patient-Reported Measures}

As stated previously, patients with MCCs have highly variable, complex, and often subjective needs. Hence, it can be difficult to identify problems, trace changes over time, compare or integrate information acquired by multiple providers, and set performance standards. Patientreported measures may be a useful tool to support these functions. Patient-reported measures are standardized quantitative data collection instruments used to obtain reports from patients about their health status or experiences receiving care services. Since they can be used in the collection of content specific to the perspectives and experiences of patients, it has been argued that they may be particularly useful for promoting and evaluating patientcentered care $[17,18]$.

The rationale espoused for using patient-reported measures as tools for these ends is often based on the subject matter they can be used to assess (patient perspectives and experiences) and the fact that the information comes directly from patients. However, it has been noted that, beyond the general content and method of data acquisition, more legitimate claims of patient centeredness depend on the meaningful involvement of patients in the development of instruments. Patient involvement in the development process has been argued to help ensure the content validity, psychometric properties, and feasibility of the instruments, and that patients' experiences with illness are adequately reflected $[9,19]$.

Here we focus on another element of patient-reported measures that is implied in statements about their value for patient-centered care: they are standardized and quantitative. This simply means that the same measure is used for multiple instances of data collection (regardless of whether these instances are distributed among multiple individuals, or over multiple points in time for the same individual), and that all data must be quantified. These data sources serve a central function in patient-centered reform: to produce data on patient perspectives and experiences (inherently subjective, variable, and complex) that is consistent, reliable, and simple enough to support decision making.

The two broad categories of patient-reported measures are patient-reported outcome measures (PROMs) and patient-reported experience measures (PREMs).

\subsection{Patient-Reported Outcome Measures (PROMs)}

PROMs contain questions on experiential domains of health. PROM domains include a wide variety of illness symptoms, treatment side effects, functionality, and quality of life [7-9, 20, 21]. PROMs have both clinical and evaluative applications. Broadly speaking, they can be classified as either generic or condition specific. Generic PROMs contain items applicable to a wide variety of patients. This makes them suitable for comparisons across conditions, but less suitable for detecting details specific to particular patient groups and changes in individuals' health over time. Condition-specific PROMs contain details applicable only to a particular condition or set of circumstances. They are thus appropriate for screening within a relatively homogenous diagnostic group, and detecting changes in individuals' health status over time, but do not yield data relevant to more general populations [17, 22].

PROMs have primarily been used in research settings including clinical trials $[19,23]$, but are also used as clinical management tools [17]. Given their origins as instruments for epidemiological and evaluative applications, most available PROMs may not be suitable for use in the clinical management of individual patients. It is essential, in selecting tools for this purpose that they be relevant to the provider's case-mix, and capable of identifying clinically significant changes in individual health status [17, 22, 24]. While there are a number of issues regarding the development and content of PROMS [19, 25], and the role of patient involvement in claims of patient-centeredness [9], our focus here is on specifying the various types of application. 


\section{PROM Uses:}

Clinical Management of Individual Patients

- Systematic screening of issues that are otherwise prone to go undetected or unassessed by clinicians [17, 20].

- Monitoring: data collected on a single patient is fed back to that patient and his or her provider longitudinally; allows patient and provider to reflect on treatment progress and act accordingly [17, 20, 21].

Facilitating Multi-Disciplinary Care

- Gives diverse providers a structured method to document patient problems and may provide a common reference point, facilitating discussion and planning $[20,21]$.

Evaluation

- Assessing and comparing outcomes achieved by different providers, to support consumer choice or inform funding decisions [17].

- Assessing the effectiveness of system-wide or routine practices and treatments to identify areas needing improvement [17, 20].

\subsection{Patient-Reported Experience Measures (PREMs)}

PREMs contain questions regarding patients' experiences receiving care. PREMs often ask questions about the accessibility of services, the quality of provider communication, care coordination and continuity, patient involvement in care decisions and management, and aspects of the humanity of care, such as respect and empathy [26-30].

PREMs are used primarily for the evaluation of services and to drive service improvement. As with outcome measures, the involvement of patients in the development of instruments, and the extent to which available instruments can be used to assess the most important aspects of care, are of crucial importance [31]. ${ }^{1}$ However, here we focus on identifying the types of applications in which PREMs are used.

PREM Uses:

Evaluation

- Assessing providers and organizations with respect to mandated patient experience care standards [32].

- Deciding on the distribution of public funds [33].

- Defining and assessing experience-based or patientcentered accreditation standards [27, 34].

- Providing experience data to consumers to support informed choice of providers [35].

\footnotetext{
${ }^{1}$ For a discussion of patient involvement in the design and evaluation of health services, see Kreindler [14].
}

\section{Context and Consequences}

Systematic reviews have been conducted examining the influences of using patient-reported measures on relatively narrowly defined aspects of care. Modest success has been reported in the effects of using PROMs, for example, in routine practice on certain communication practices, and the detection of mental health conditions [36, 37]. Despite the tentative promise of these findings, the potential of patient-reported measures as tools for fostering a patientcentered approach to care can only be maximized by considering the processes through which they are meant to affect change. Greenhalgh et al. [38] have noted the importance, in the evaluation of PROM initiatives, of specifying and testing for the presence of factors theorized or known to be necessary to produce desired outcomes. As part of this approach, the authors emphasize the careful examination of implementation contexts, and assessment of their concordance with intervention theory.

We argue that, in addition to careful attention to context, the introduction and evaluation of patient-report initiatives should be accompanied by continual vigilance for unplanned effects on patient care. In the following sections, we identify some likely contextual influences on the manner in which initiatives will influence existing care practices. Then, instead of focusing on how initiatives and contexts interact to produce intended outcomes, we consider how this interaction may produce unintended consequences.

\subsection{Contextual Consideration \#1: Overburdened Practices and Challenging Case-Mixes (Direct-Care Level)}

Prioritizing and enacting high-quality dialogue between patients and providers requires time and human resources. Yet many primary care practices are understaffed, underresourced, and struggling to meet competing demands [39]. Studies have shown that primary care providers do not have enough time to adhere to clinical practice guidelines for chronic conditions. Given the challenges associated with interpreting multiple changing, often subjectively defined, symptoms, the difference between actual and optimal consultation time for MCC patients stands to be even greater. Moreover, treatment guidelines, designed to help clinicians manage the decision-making process, are usually based on single-disease samples, and are of limited applicability to MCC patients. This places greater onus on the qualitative dimension of consultation as a means of understanding and addressing the problems of unique and complex individuals [1]. Yet this process takes time and provider attention, resources that, as mentioned, are often in short supply. 
5.2 Contextual Consideration \#2: Fragmented Information Technology (IT) Systems

(Health-Systems Level)

Since modern health systems consist of diverse and geographically dispersed providers and organizations, it is necessary to use information technology (IT) to share data and develop multi-disciplinary care plans. The process of developing integrated care plans requires compatible technology and common methods for entering and coding data. However, many healthcare IT systems today are characterized by incompatible hardware and software, and lack of common nomenclature and coding structures. Patient-reported measures are an increasingly common form of information within a vast sea of data [10, 40]. Without proper precautions and planning, the addition of more patient-reported data may contribute to, rather than alleviate, confusion in developing effective and shared protocols.

\subsection{Unintended Consequences}

By measuring patient health, illness, and healthcare performance using standardized data capture, patient-reported measures focus on precisely defined, measurable aspects of health and healthcare. Policy mechanisms, such as the mandatory use of PROMs [24] and financial or accreditation incentives for achieving patient experience benchmarks [27, 34, 41], prompt organizations and providers to change their behaviours and policies. In a time- and resource-constrained environment, this means that less time and resources go into other priorities [39], including some that may be as or more vital to the provision of care for patients. In the UK for example, Campbell et al. [34] argue that a nationwide initiative tying financial incentive to rapid primary care access, as measured by a single-item PREM, may have led to a widespread decrease in providerspecific continuity in primary care. By encouraging providers to ensure rapid access to any provider within their organization as a means of ensuring rapid access, they argue, organizations may have sacrificed continuous access to the same provider, an aspect of care that was not incentivized. We have argued that provider-specific continuity is a central aspect of a patient-centered approach for patients with MCCs because of the need to make sense of each individual's complex needs over time.

In addition to influencing organizational practices, the very act of using standardized assessment tools directly impacts what happens during patient-provider consultation. Providers participating in the above-mentioned UK initiative suggested that the emphasis on "ticking boxes" distracted them from patient-led consultations and listening to patient concerns [41]. More generally, DeLusignan and
Chan [42] suggest that the use of standardized computer assessment tools during patient consultation can lead to a narrowing of focus on easily quantifiable data, the neglect of vague complaints, social diagnoses, and physical manifestations of underlying clinical problems. The possibility of these impacts requires consideration, given the importance of a dialogic, iterative approach to consultation for patients with MCCs.

The wide-scale use of patient-reported measures and other standardized assessment tools also affects, for better or for worse, the information foundations on which multidisciplinary care protocols are meant to be based. Evidence regarding the widespread introduction of computer physician order entry (CPOE) systems in the USA provides some insight on this issue. While CPOE systems have led to some modest reductions in medication errors, they have also been linked to several new errors. In the case of patient-reported measures, which require input from both providers and patients, the potential for error would seem even greater. Making data available to providers that is erroneous, contextually inappropriate, or impossible to reconcile with other sources compounds, rather than mitigates, the challenges associated with formulating a coherent, adaptive picture of patient needs and progress. Where data are entered and accessed via a large and highly connected IT network, the consequences stand to be correspondingly vast and varied [43].

\section{Conclusions}

Great attention is placed in healthcare, understandably, on solutions. However, it is always worth considering the possibility that a proposed solution may prompt unanticipated effects. This is particularly important where health problems are hard to understand and predict, as is the case with treatment provision for patients with MCCs. Becoming knowledgeable about problems and familiar with the contexts in which they are addressed is essential for solving them and for preventing unintended harms. However, beyond this, maintaining a degree of skepticism about any proposed solution is generally warranted.

We have highlighted the time-constrained, resourcelimited nature of contemporary healthcare settings and the fragmentation of provider-provider IT systems. We have argued that these factors are likely to influence the impact that the use of patient-reported measures has on the key care processes of patient-provider consultation and formulation of individualized multi-disciplinary care protocols. Subsequently, we argued that operationalizing patient-reported measures by mandating or incentivizing their use may result in unanticipated and potentially harmful effects to these key activities. 
In this commentary, we have attempted to stimulate discussion and encourage critical reflection concerning the use of patient-reported measures. In doing this, we have no intention of categorically rejecting initiatives that employ them, nor of discouraging those who want to work on the problems associated with MCC care. To the contrary, we believe that patient-reported measures can be of great value in supporting patient-centered care for these patients. Rather, our intention has been to encourage critical thought and discussion on these topics, because we believe this to be essential to the success of patient-centered initiatives in a complex and unpredictable world.

Acknowledgments Funding for this study was provided by the Ontario Ministry of Health and Long-term care through the Health System Performance Research Network at the University of Toronto. The views reflected in this manuscript are those of the research team and not the funder.

\section{Conflict of interest None.}

Author contributions Daniel Miller led the conception, preparation, and revision of this manuscript. Carolyn Steele Gray, Kerry Kuluski and Cheryl Cott were involved in its conception, preparation and revision. All authors approved the final submitted version of the manuscript.

Open Access This article is distributed under the terms of the Creative Commons Attribution Noncommercial License which permits any noncommercial use, distribution, and reproduction in any medium, provided the original author(s) and the source are credited.

\section{References}

1. Upshur RE, Tracy S. Chronicity and complexity: is what's good for the diseases always good for the patients? Can Fam Physician. 2008;54(12):1655-8 pii:54/12/1655.

2. Freid VM, Bernstein AB, Bush MA. Multiple chronic conditions among adults aged 45 and over: trends over the past 10 years. NCHS Data Brief. 2012;100:1-8.

3. Ministry of Health and Long-Term Care. Preventing and Managing Chronic Disease: Ontario's Framework: Ministry of Health and Long-Term Care; 2007.

4. Boyd CM, Fortin M. Future of multimorbidity research: how should understanding of multimorbidity inform health system design? Public Health Rev. 2010;32(2):451-74.

5. Barnett K, Mercer SW, Norbury M, Watt G, Wyke S, Guthrie B. Epidemiology of multimorbidity and implications for health care, research, and medical education: a cross-sectional study. Lancet. 2012;380(9836):37-43.

6. Salisbury C. Multimorbidity: redesigning health care for people who use it. Lancet. 2012;380(9836):7-9. doi:10.1016/S01406736(12)60482-6.

7. Leidy NK, Vernon M. Perspectives on patient-reported outcomes: content validity and qualitative research in a changing clinical trial environment. Pharmacoeconomics. 2008;26(5):363-70.

8. United States Food and Drug Administration (FDA). Guidance for industry: Patient-reported outcome measures: use in medical product development to support labeling claims: draft guidance.
Health Qual Life Outcomes. 2006;4:79. doi:10.1186/1477-75254-79.

9. Trujols J, Portella MJ, Iraurgi I, Campins MJ, Siñol N, Cobos JPDL. Patient-reported outcome measures: are they patient-generated, patient-centred or patient-valued? J Ment Health. 2013;22(6):555-62. doi:10.3109/09638237.2012.734653.

10. Institute of Medicine. Crossing the quality chasm: a new health system for the 21st Century. Washington, DC: National Academic Press; 2001.

11. Frampton S, Guastello S, Brady C, Hale M, Horowitz S, Bennet Smith $\mathrm{S}$, et al. Patient-centered care improvement guide. Planetree; 2008.

12. Stewart M, Brown BB, Weston WW, McWhinney IR, McWilliam CL, Freeman TR. Patient-centered medicine: transforming the clinical method. 1st ed. Oxon: Sage Publications, Inc.; 1995.

13. Hudon C, Fortin M, Haggerty J, Loignon C, Lambert M, Poitras ME. Patient-centered care in chronic disease management: a thematic analysis of the literature in family medicine. Patient Educ Couns. 2012;88(2):170-6. doi:10.1016/j.pec.2012.01.009.

14. Kreindler SA. Patient involvement and the politics of methodology. Can Public Adm. 2009;52(1):113-24. doi:10.1111/j.17547121.2009.00062.x.

15. Klein G, Moon B. Making sense of sensemaking 1: alternative perspectives. IEEE Intell Syst. 2006;21(4):70-3.

16. Klein G, Moon B. Making sense of sensemaking 2: a macrocognitive model. IEEE Intell Syst. 2006;21(5):88-92.

17. Black N. Patient reported outcome measures could help transform healthcare. BMJ. 2013;346:f167. doi:10.1136/bmj.f167.

18. Jenkinson $C$, Coulter A, Bruster S. The picker patient experience questionnaire: development and validation using data from inpatient surveys in five countries. Int $\mathrm{J}$ Qual Health Care. 2002;14(5):353-8. doi:10.1093/intqhe/14.5.353.

19. Staniszewska S, Haywood KL, Brett J, Tutton L. Patient and public involvement in patient-reported outcome measures: evolution not revolution. Patient. 2012;5(2):79-87. doi:10.2165/ 11597150-000000000-00000.

20. Greenhalgh J. The applications of PROs in clinical practice: what are they, do they work, and why? Qual Life Res. 2009;18(1): 115-23. doi:10.1007/s11136-008-9430-6.

21. Snyder C, Aaronson N, Chouclair A, Elliott T, Greenhalgh J, Halyard M, et al. Implementing patient-reported outcomes assessment in clinical practice: a review of the options and considerations. Qual Life Res. 2012;21(8):1305-14. doi:10.1007/ s11136-011-0054-x.

22. Hogg FRA, Peach G, Price P, Thompson MM, Hinchliffe RJ. Measures of health-related quality of life in diabetes-related foot disease: a systematic review. Diabetologia. 2012;55(3):552-65. doi:10.1007/s00125-011-2372-5.

23. Bryan SBJ, Dalzell K, Davis J, Dawes M, Doyle-Waters MM, Lewis $\mathrm{S}$, et al. What are the most effective ways to measure patient health outcomes of primary health care integration through PROM (patient reported outcome measurement) instruments? Vancouver: Centre for Clinical Epidemiology \& Evaluation; 2013.

24. Wolpert M. Uses and abuses of patient reported outcome measures (PROMs): potential iatrogenic impact of proms implementation and how it can be mitigated. Adm Policy Ment Health. 2014;41(2):141-5. doi:10.1007/s10488-013-0509-1.

25. Frei A, Svarin A, Steurer-Stey C, Puhan MA. Self-efficacy instruments for patients with chronic diseases suffer from methodological limitations-a systematic review. Health Qual Life Outcomes. 2009;7:86. doi:10.1186/1477-7525-7-86.

26. Lehrman WG, Elliott MN, Goldstein E, Beckett MK, Klein DJ, Giordano LA. Characteristics of hospitals demonstrating superior performance in patient experience and clinical process measures of care. Med Care Res Rev. 2010;67(1):38-55. 
27. Scholle SH, Vuong O, Ding L, Fry S, Gallagher P, Brown JA, et al. Development of and field test results for the CAHPS PCMH Survey. Med Care. 2012;50 Suppl.:S2-10.

28. Williams A, Kristjanson L. Emotional care experienced by hospitalised patients: development and testing of a measurement instrument. J Clin Nurs. 2009;18(7):1069-77.

29. Schäfer WLA, Boerma WGW, Kringos DS, De Ryck E, Greß S, Heinemann S, et al. Measures of quality, costs and equity in primary health care instruments developed to analyse and compare primary care in 35 countries. Qual Prim Care. 2013;21(2):67-79.

30. Picker Institute Europe. Inpatient Questionnairre. 2009.

31. International Alliance of Patients' Organizations (IAPO). Patient Centred Healthcare Indicators review 2012.

32. Care Quality Commission. About us: What we do and how we do it. 2012. http://www.cqc.org.uk/sites/default/files/media/documents/ 201202-cqc-about_us.pdf. Accessed 20 Nov 2013.

33. Clark C. 15 HCAHPS Buzzwords Every Provider Should Know. Health Leaders Media Council. 2011. http://www.healthleaders media.com/page-1/PHY-268393/15-HCAHPS-Buzzwords-EveryProvider-Should-Know. Accessed 30 Oct 2013.

34. Campbell SM, Reeves D, Kontopantelis E, Sibbald B, Roland M. Effects of pay for performance on the quality of primary care in England. N Engl J Med. 2009;361(4):368-78.

35. Goldstein E, Cleary PD, Langwell KM, Zaslavsky AM, Heller A. Medicare managed care CAHPS $\AA$ : a tool for performance improvement. Health Care Financ Rev. 2001;22(3):101-7.

36. Marshall S, Haywood K, Fitzpatrick R. Impact of patient-reported outcome measures on routine practice: a structured review. J Eval Clin Pract. 2006;12(5):559-68.
37. Valderas JM, Kotzeva A, Espallargues M, Guyatt G, Ferrans CE, Halyard MY, et al. The impact of measuring patient-reported outcomes in clinical practice: a systematic review of the literature. Qual Life Res. 2008;17(2):179-93.

38. Greenhalgh J, Long AF, Flynn R. The use of patient reportd outcome measures in clinical practice: lack of impact or lack of theory? Soc Sci Med. 2005;60:833-43.

39. Estabrooks PA, Boyle M, Emmons KM, Glasgow RE, Hesse BW, Kaplan RW, et al. Harmonized patient-reported data elements in the electronic health record: Supporting meaningful use by primary care action on health behaviors and key psychosocial factors. J Am Med Inform Assoc. 2012;19(4):575-82.

40. Black AD, Car J, Pagliari C, Anandan C, Cresswell K, Bokun T, et al. The impact of eHealth on the quality and safety of health care: a systematic overview. PLoS Med. 2011;8(1):e1000387. doi:10.1371/journal.pmed.1000387.

41. Gillam SJ, Niroshan Siriwardena A, Steel N. Pay-for-performance in the United Kingdom: impact of the quality and outcomes framework-a systematic review. Ann Fam Med. 2012;10(5):461-8. doi:10.1370/afm.1377.

42. De Lusignan $S$, Chan $T$. The development of primary care information technology in the United Kingdom. J Ambul Care Manage. 2008;31(3):201-10.

43. Strom BL, Schinnar R. Evaluating health information technology's clinical effects. LDI Issue Brief. 2011;16(4):1-4. 